\title{
Antimicrobial and Antioxidant Activities of Zingiber officinale (Ginger) and Alpinia officinarum (Galangal)
}

\author{
Gulcin Alp Avci' ${ }^{1}$ Emre Avci $^{1}$ (D) Gizem Ozluk Cilak ${ }^{2}$ Sule Coskun Cevher ${ }^{3}$ (D) \\ ${ }^{1}$ Hitit University, Department of Molecular Biology and Genetics, Corum, Turkey \\ ${ }^{2}$ Hitit University, Department of Food Engineering, Corum, Turkey \\ ${ }^{3}$ Gazi University, Department of Biology, Ankara, Turkey
}

\section{A BS T R AC T}

$\mathrm{T}$ he use of spices in the treatment of health problems has been a tradition in the world since early ages. Alpinia officinarum Hance (galangal) and Zingiber officinale Roscoe (ginger) are aromatic plants, enriched with bioactive compounds providing the usage for their therapeutic properties. In this study, the ethanol and water (ultra-pure) extracts of galangal and ginger are used to determine the antimicrobial and antioxidant properties. Antioxidant effect was evaluated based on total antioxidant status performed by automated colorimetric measurement method and DPPH free radicals scavenging effects done by spectrophotometric method; whilst antimicrobial effect was observed on Enterecoccus faecalis, Escherichia coli, Staphylococcus aureus, Pseudomonas aeroginosa and Candida albicans. The results indicate that, both ginger and galangal extracts demonstrated effective antimicrobial and antioxidant properties. Their consumption may restrain the oxidation and prevent or delay the degenerative diseases as well as their extracts can be used as antimicrobial agents.

Keywords:

Alpinia officinarum Hance; Zingiber officinale roscoe; Galangal; Ginger; Antimicrobial; Antioxidant; Free radical scavenging.

\section{INTRODUCTION}

$\mathrm{T}$ he use of spices in the treatment of health problems has been a tradition in the world since early ages. As consumer preferences shift to natural products, the use of spices and natural aromatic plants as antioxidants and antimicrobials instead of synthetic food additives has been back on the agenda recently. The importance of spices and aromatic plants has increased due to the side effects of synthetic drugs and the fact that bacteria can easily develop resistance to these synthetic drugs [1-4].

Alpinia officinarum Hance (galangal) and Zingiber officinale Roscoe (ginger) are aromatic plants, both belong to Zingiberaceae family, rhizomes of which are used as spice [5]. Galangal is enriched with bioactive components providing the usage as medicine mostly in Far East countries in many diseases such as cold, bronchitis, stomach ache, diabetes, ulcer, abdominal swelling, diarrhea, vertigo, neupathia, rheumatoid arthritis and inflammatory bowel diseases etc. [6, 7]. Ginger is well known for its bioactive constituents, revealing antimicrobial, antifungal and antioxidant effects. It is widely used for its anti-inflammatory and anti-tumorigenic functions in addition to aiding properties of cholesterol lowering and digestive problems [8-10].

These therapeutic properties of spices derive from their chemical compounds that lead to have antioxidant and antimicrobial effects. Antimicrobial activity varies depending on the type and concentration of the spice as well as microbial density and diversity. Antimicrobial effect is mostly provided by phenolic and terpenoid compounds containing hydroxyl group which has the inhibitory effect via destroying the phospholipid layer of cell membrane. Thus, the increased permeability of cell membrane causes all the cell content comes out. Spices are effective at all stages of microbial growth such as lengthening the lag phase or decreasing the growth rate in exponential phase and the total reduction in cell count $[5,11]$. The antimicrobial activity of ginger was studied on some microorganisms such as Pseudomonas aeruginosa, Staphylococcus aureus, Salmonella Typhimurium and Listeria monocytogenes; whereas Bacillus subtilis, Candida albicans, Enterococcus faecalis, Enterobacter aerogenes, Enterococcus durans, Enterococcus faecium, Escherichia coli, Klebsiella pneumoniae, Listeria innocua, Pseudomonas fluorescence, Salmonella enteritidis, Salmonella infantis, Salmonella kentucky 
and Staphylococcus epidermidis were the microorganisms studied for galangal antimicrobial activity $[10,12,13]$.

Oxidation is a chemical reaction that can produce free radicals and thus leads to chain reactions that can damage the cells of organism. Antioxidant substances prevent degenerative diseases via restraining oxidation. The oxidation blocking path of spices is provided by phenolic compounds whereby free radical scavenging, compounding with metal ions and preventing the formation of singlet oxygen. It was observed that the higher the number of hydroxyl groups in the aromatic ring are, the higher antioxidant activity of the spice is $[5,14-16]$. It has been proposed to use free radical scavenging, one of the antioxidant mechanisms, for therapeutic purposes. Radical scavengers may directly affect peroxide radicals and end the oxidation chain reactions [16].

It was shown by the studies that the extractive value of the spices shows difference according to the solvent used [9, 16]. In this study, the ethanol, and water (ultra-pure) extracts of Alpinia officinarum Hance (galangal) and Zingiber officinale Roscoe (ginger) are used to determine the antimicrobial and antioxidant properties based on total antioxidant status and DPPH free radicals scavenging effects.

\section{MATERIAL AND METHODS}

\section{Samples}

Zingiber officinale (Ginger) and Alpinia officinarum (Galangal) samples were obtained commercially.

\section{Preparation of Extracts}

The preparation of extracts is schematized in Fig. 1. After extraction procedure samples were stored at $4^{\circ} \mathrm{C}$.

\section{Determination of Antimicrobial Activity}

\section{Microorganisms}

Antimicrobial activities of extracts prepared with ethanol and water were used against five microorganisms. In order to represent gram positive bacteria, S. aureus ATCC 25923 and E. faecalis ATCC 29212 are used; whereas E. coli ATCC 25922 and P. aeroginosa (ATCC 27853) were used to represent gram negative bacteria. In addition, one fungus C. albicans ATCC 10231 were also used to determine the antimicrobial and antioxidant effects.

\section{Agar-well and Disc Diffusion Method}

The antimicrobial activity was determined by diffusion methods (disc and agar-well). Bacterial cultures were grown at $37^{\circ} \mathrm{C}$ for $24 \mathrm{~h}$ and C. albicans was grown at $25^{\circ} \mathrm{C}$ for $48 \mathrm{~h}$. Suspensions were adjusted according to McFarland 0.5 standard. $100 \mu \mathrm{l}$ microbial cultures added with Mueller-Hinton Agar (MHA) in Petri plates. 6-mm agarwell opened or disc prepared using paper (Watmann no. 4) placed on MHA. Extracts $(15 \mu \mathrm{L})$ were inoculated in wells or on discs. Then, plates were incubated for 24-48 hours. After incubation, diameter of the inhibition zone was measured.

\section{Determination of Antioxidant Activity}

\section{DPPH Radical Scavenging Assay}

Radical scavenging activity was determined by a spectrophotometric method developed by Blois (1958) and Khalaf et al. (2008) [18, 19]. Tests were repeated three times. Ascorbic acid was used as positive control. Inhibition ratio (\%) of free radical was calculated according to formula given below where IDA is the inhibition of DPPH activity:

$$
I D A(\%)=\frac{(\text { Ablank }- \text { Asample })}{\text { Ablank }} \times 100
$$

\section{Total Antioxidant Status assay}

The extracts were examined in terms of total antioxidant

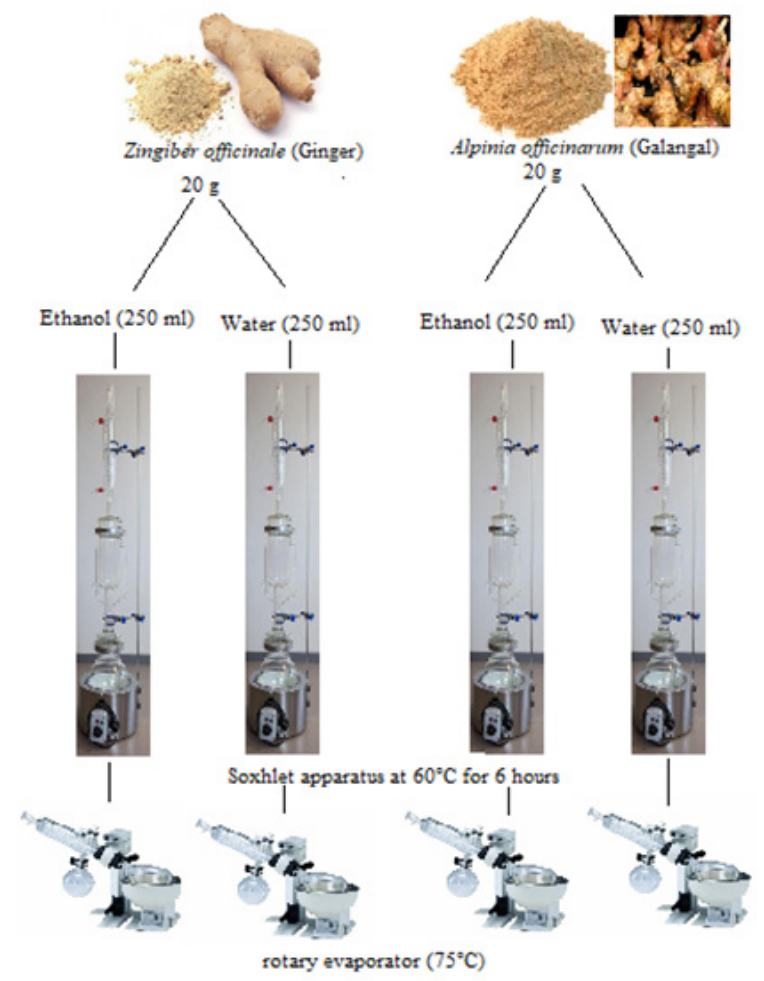

Figure 1. Extracts' preparation. 


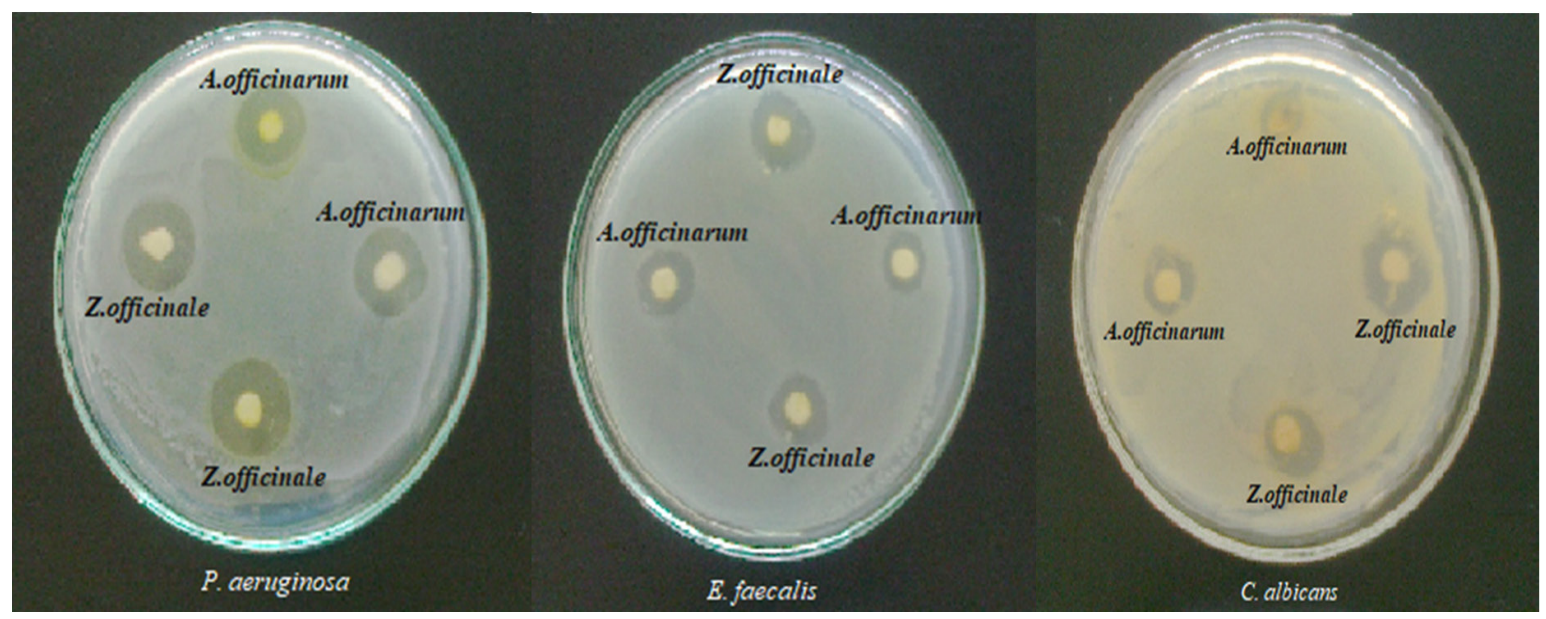

Figure 2. Antimicrobial effects on some pathogen strains of Z. officinale and A.officinarum by diffusion method.

status (TAS) by a colorimetric method developed by Erel (2004). In this method, Fenton reaction is used to produce hydroxyl radical and bright yellowish-brown dianisyl radical is produced by reacting with colorless substrate o-dianisidine. Results were expressed as micromolar Trolox equivalents per liter ( $\mu \mathrm{mol}$ Trolox Eq/L).

\section{Statistical analysis}

Statistical analysis of this study was performed on the data by SPSS 22.0 (SPSS Inc., Chicago). The statistical significance determined at $\mathrm{p}<0.05$ in this study. All results were expressed as means \pm standard deviations (SD).

\section{RESULTS AND DISCUSSION}

\section{Determination of antimicrobial and antifungal activity}

Looking at the data observed in Table 1 and Fig. 2, it is avowable that ginger had slightly higher antimicrobial effect on all species than galangal $(\mathrm{P}<0.05)$. The antimicrobial effect is considered as week $(<12 \mathrm{~mm})$, moderate (12 to $\leq 20 \mathrm{~mm}$ ) and strong ( $\geq 20 \mathrm{~mm}$ ) according to Pillai et al [20]. Ethanol extracts of the plants frankly made higher antimicrobial effect than distilled water extracts. Ethanol extracts showed significant antibacterial acti-

Table 1. Antimicrobial activities of Ginger and Galangal extracts by Disc diffusion method and Agar diffusion method.

\begin{tabular}{|c|c|c|c|c|}
\hline & \multicolumn{4}{|c|}{ Strains (Inhibition zone diameter, $\mathrm{mm}$ ) } \\
\hline & \multicolumn{2}{|c|}{ Zingiber officinale (Ginger) } & \multicolumn{2}{|c|}{ Alpinia officinarum (Galangal) } \\
\hline \multicolumn{5}{|c|}{ Disc diffusion method } \\
\hline S. aureus & $13 \cdot 5 \pm 1.5$ & $11.0 \pm 1.0$ & $12.5 \pm 2.0$ & $10.4 \pm 2.2$ \\
\hline E. faecalis & $13.2 \pm 2.8$ & $10.2 \pm 1.8$ & $12.0 \pm 1.5$ & $9 \cdot 7 \pm 1.6$ \\
\hline$P$. aeruginosa & $15.8 \pm 1.2$ & $11.6 \pm 1.4$ & $14.1 \pm 1.9$ & $11.2 \pm 1.5$ \\
\hline E. coli & $8.4 \pm 0.6$ & ND & $7.1 \pm 0.9$ & $N D$ \\
\hline C. albicans & $11.2 \pm 1.3$ & $9 \cdot 4 \pm 0.6$ & $10.8 \pm 1.2$ & $8.8 \pm 0.7$ \\
\hline \multicolumn{5}{|c|}{ Agar diffusion method } \\
\hline S. aureus & $15 \cdot 2 \pm 1.0$ & $12.0 \pm 1.0$ & $13.2 \pm 1.8$ & $11.4 \pm 1.6$ \\
\hline E. faecalis & $14.8 \pm 0.5$ & $11.6 \pm 1.5$ & $13 \cdot 5 \pm 1.5$ & $10.8 \pm 1.2$ \\
\hline P. aeruginosa & $18.5 \pm 2.8$ & $14.0 \pm 2.0$ & $16.6 \pm 2.6$ & $13 \cdot 7 \pm 2.7$ \\
\hline E. coli & $10.2 \pm 1.4$ & $6.0 \pm 1.5$ & $9.1 \pm 0.6$ & $N D$ \\
\hline C. albicans & $13.2 \pm 2.2$ & $11.6 \pm 1.4$ & $12.1 \pm 1.8$ & $10.2 \pm 0.8$ \\
\hline
\end{tabular}




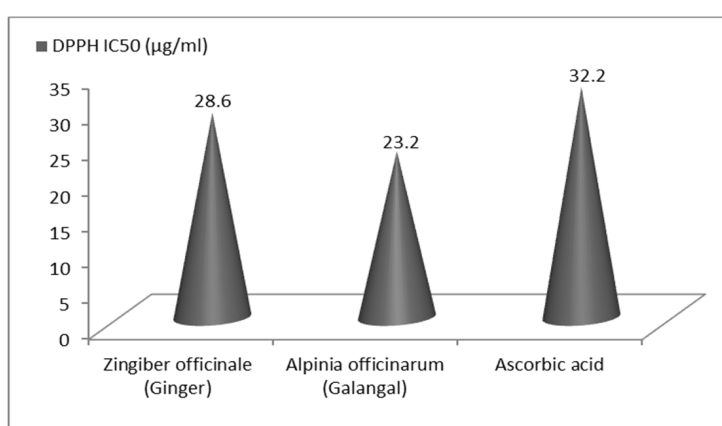

Figure 3. DPPH radical scavenging of Ginger and Galangal ethanol extracts.

vity against all tested bacterial strains $(\mathrm{P}<0.05)$. Ginger and galangal extracts showed week antimicrobial effect on $E$. coli whereas the ethanol extracts of both plants showed moderate antimicrobial effect on $S$. aureus, $E$. faecalis and $P$. aeruginosa $(\mathrm{P}<0.05)$. E. coli was the most durable strain whilst $P$. aeruginosa is the most unstable one. The antifungal activity of both ginger and galangal extracts were found to be week against $C$. albicans in disc diffusion; moderate in agar diffusion method.

The inhibition zones were found larger in agar diffusion method than those in disc diffusion method. The difference can easily be explained by the reason that the extracts are not absorbed well in discs as in agar diffusion method. However, standards are available for disc diffusion method, such as the type of media used, agar percentage, and thickness of media, which lead no ambiguity.

Srividya et al. [21] made experiments on hydro alcoholic extract by hot and cold maceration and methanol extract by percolation process of galangal antimicrobial effect. They found that all three extracts showed moderate antimicrobial activity against the $B$. cereus, $S$. aureus, $P$. aeuroginosa, $E$. coli but no antifungal activity against $A$. niger and $C$. albicans. This can be concluded by the reason that extraction method can show an alteration in the result because in the present study antifungal activity was observed. Sharef et al. [22] studied the antimicrobial effect of ginger methanolic extract comparing with the standard

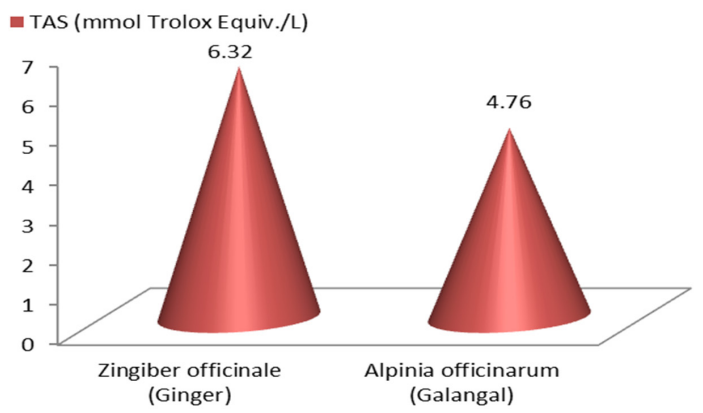

Figure 4. The antioxidant activities of Ginger and Galangal ethanol extracts. antibiotics on Escherichia coli, Pseudomonas aerogenosa, Proteus mirabilis, Staphylococcus aureus and Klebsiella pneumonia by using the agar diffusion method. They have revealed that methanolic extracts of ginger showed stronger antimicrobial effect compared to Streptomycin, Rifambin and Cefotoxime. This study can clearly help interpreting the present results of ginger and galangal extracts have effective antimicrobial properties than the standard antibiotics, though their moderate and week inhibition zones.

\section{Determination of Antioxidant Activity as DPPH Radical Scavenging and Total Antioxidant Status}

The antioxidant properties were determined based on DPPH free radicals scavenging effects and total antioxidant status. DPPH radical scavenging and antioxidant activities of ginger and galangal ethanol extracts are presented in Fig. 3 and Fig. 4. The effect of antioxidants on DPPH derives from their hydrogen donating ability [23]. The results obtained showed that the ethanol extracts of ginger and galangal radical scavenging abilities were nearly the same when compared to ascorbic acid. The lower IC50 value is, the higher radical scavenging effect is; therefore, galangal was found to have higher scavenging ability than ginger. Khalaf et al. [18] found similar results in their own study when comparing the radical scavenging effect of Zingiber officinale Roscoe with ascorbic acid. These results can be interpreted as ginger and galangal have antioxidant effect as much as ascorbic acid has. Scavenging free radicals are proposed to be used for therapeutic expectations [16]. Ghasemzadeh et al [23] found in their study that methanol extracts of ginger have good free radical scavenging ability comparing with alpha-tocopherol. Köse et al. [24] found high but different DPPH radical scavenging values of water, water-ethanol and ethanol extracts of galangal comparing with BHA, $\mathrm{BHT}$, trolox and alpha-tocopherol. These studies indicate that the extract chemical as well as the compared material makes disparate effect on the results.

In this study, the total antioxidant status was found to be higher in ginger than galangal in contrast with the scavenging effect. Çiftçi et al. [25] expressed their study results in beneficial usage of ginger on total antioxidant status which they used the Erel [19] method to analyse. No total antioxidant status essays of galangal has been recorded in researches, hence, our study is expected to have contribution to literature.

\section{CONCLUSION}

The present study demonstrated that both ginger and galangal extracts have effective antimicrobial and antioxidant properties. Their consumption may restrain the 
oxidation and prevent or delay the degenerative diseases. Discovering novel antimicrobial agents have become important against the microbial infections. This study shows that ginger and galangal extracts can be used as antimicrobial agents.

\section{References}

1. Çoban Ö.E., Patır B. Use of Some Spices and Herbs Antioxidant Affected in Foods. Electronic Journal of Food Technologies, 5(2): 7-19. 2010.

2. Bérdy J. Thoughts and facts about antibiotics: Where we are now and where we are heading. The Journal of Antibiotics. 65: 385-395, 2012 .

3. Iyer A, Panchal S, Poudyal H, Brown L. Potential Health Benefits of Indian Spices in the Symptoms of the Metabolic Syndrome: A Review. Indian Journal of Biochemistry and Biophysics, 46 (6):467 $81,2009$.

4. Martins IJ. Indian Spices and Biotherapeutics in Health and Chronic Disease. Health, 10 (4): 374-380, 2018.

5. Akgül, A. Baharat Bilimi ve Teknolojisi. Gida Teknolojisi Dergisi Yayınlar1, No:15, 450 s., Ankara, 1993.

6. Honmore VS, Kandhare AD, Kadam PP, Khedkar VM, Sarkar D, Bodhankar SL, Zanwar AA, Rojatkar SR, Natu AD. Isolates of Alpinia officinarum Hance as COX-2 inhibitors: Evidence from anti-inflammatory, antioxidant and molecular docking studies. International Immunopharmacology, 33: 8-17, 2016.

7. Basri AM, Taha H, Ahmad N. A Review on the Pharmacological Activities and Phytochemicals of Alpinia officinarum (Galangal) Extracts Derived from Bioassay-Guided Fractionation and Isolation. Pharmacogn Rev. 11(21): 43-56, 2017.

8. Srinivasan K. Ginger rhizomes (Zingiber officinale): A spice with multiple health beneficial potentials. PharmaNutrition, 5 (1): 18-28, 2017.

9. Arawande JO, Akinnusotu A, Alademeyin JO. Extractive Value and Phytochemical Screening of Ginger (Zingiber officinale) and Turmeric (Curcuma longa) Using Different Solvents. International Journal of Traditional and Natural Medicines, 8 (1): 13-22, 2018.

10. Noori S, Zeynali F, Almasi H. Antimicrobial and antioxidant efficiency of nanoemulsion-basededible coating containing ginger (Zingiber officinale) essential oil and its effect on safety and quality attributes of chicken breast fillets. Food Control, 84: 312-320, 2018.

11. Aybakır M. Investigating antimicrobial effects of spices in hurdle technology. Master Thesis. Ankara University, Graduate School of Applied Sciences, Department of Food Engineering. Ankara, 2015.

12. Azadpour M, Azadpour N, Bahmani M, Hassanzadazar H, Kopaei
MR, Naghdi N. Antimicrobial effect of Ginger (Zingiber officinale) and mallow (Malva sylvestris) hydroalcholic extracts on four pathogen bacteria. Der Pharmacia Lettre, 8 (1):181-187, 2016.

13. Özkinali S, Şener N, Gür M, Güney K, Olgun Ç. Antimicrobial Activity and Chemical Composition of Coriander\&Galangal Essential Oil. Indian Journal of Pharmaceutical Education and Research. 51 (3): 221-224, 2017.

14. Baublis AJ, Clydesdale FM, Decker EA. Antioxidants in WheatBased Breakfast Cereals. Cereals Foods World. 45: 71-74, 2017.

15. Serafini M, DelRio D. Understanding the association between dietary antioxidants, redox status and disease: is the total antioxidant capacity the right tool. Redox report. 9 (3): 145-52, 2004.

16. Tohma H, Gülçin İ, Bursal E, Gören AC, Alwasel, SH, Köksal E (2017). Antioxidant activity and phenolic compounds of ginger (Zingiber officinale Rosc.) determined by HPLC-MS/MS. Journal of Food Measurement and Characterization, 11 (2): 556-566, 2017.

17. Blois MS. Antioxidant determinations by the use of a stable free radical. Nature. 29: 1199-1200, 1958.

18. Khalaf NA, Shakya AK, Al-Othman A, El-Agbar Z, Farah H. Antioxidant Activity of Some Common Plants. Turk J Biol. 32: 51-55, 2008.

19. Erel Ö. A novel automated direct measurement method for total antioxidant capacity using a new generation, more stable ABTS radical cation. Clinical Biochemistry. 37: 277- 285, 2004.

20. Pillai MK, Ismail R, Sasidharan S, Asmawi MZ. Antioxidant and antimicrobial activities of rhizome extracts from malaysian species of Alpinia galanga and Alpinia officinarum. Pharmacologyonline. 1: 366-375, 2019.

21. Srividya AR, Dhanabal SP, Misra VK, Suja G. Antioxidant and Antimicrobial Activity of Alpinia officinarum. Indian Journal of Pharmaceutical Sciences. 72 (1): 145-148, 2010.

22. Shareef HK, Muhammed HJ , Hussein HM, Hameed IH. Antibacterial Effect of Ginger (Zingiber officinale) Roscoe and Bioactive Chemical Analysis using Gas Chromatography Mass Spectrum. Oriental Journal of Chemistry. 32 (2): 817-837, 2016.

23. Ghasemzadeh A, Jaafar HZE, Rahmat A. Antioxidant Activities, Total Phenolics and Flavonoids Content in Two Varieties of Malaysia Young Ginger (Zingiber officinale Roscoe). Molecules. 15: 4324-4333, 2010.

24. Köse LP, Gülçin İ, Görenc AC, Namiesnikd J,Martinez-Ayalae AL, Gorinsteinf S. LC-MS/MS analysis, antioxidant and anticholinergic properties of galanga (Alpinia officinarum Hance) rhizomes. Industrial Crops and Products. 74: 712-721, 2015.

25. Çifci A, Tayman C, Yakut Hİ, Halil H, Çakır E, Çakır U, Aydemir S. Ginger ( Zingiber officinale ) prevents severe damage to the lungs due to hyperoxia and inflammation. Turk J Med Sci. 48 (4): 892-900, 2018 . 